\title{
Immunoglobulin A Vasculitis Masquerading as Bilateral Avascular Necrosis of the Hip
}

\author{
Davis $\mathbf{S}^{1}$, Ramahi $\mathrm{A}^{2}$, Sabha $\mathbf{M}^{2}$ and Altorok $\mathbf{N}^{1,2,3 *}$ \\ ${ }^{1}$ University of Toledo, College of Medicine and Life Sciences, USA \\ ${ }^{2}$ Department of Internal Medicine, University of Toledo, USA \\ ${ }^{3}$ Division of Rheumatology and Immunology, University of Toledo, USA
}

*Corresponding author: Nezam Altorok, University of Toledo Medical Center, 3000 Arlington Ave, Mailstop 1186, Toledo, OH

43614, USA

\section{ARTICLE INFO}

Received: 幽 February 14, 2020

Published: 慧 March 04, 2020

Citation: Davis S, Ramahi A, Sabha M, Altorok N. Immunoglobulin A Vasculitis Masquerading as Bilateral Avascular Necrosis of the Hip. Biomed J Sci \& Tech Res 26(2)2020. BJSTR. MS.ID.004325.

\section{ABSTRACT}

IgA vasculitis is a small vessel vasculitis that usually presents acutely and resolves with supportive care. However, it can cause serious complications. The etiology of IgA vasculitis and the pathophysiology of atraumatic avascular necrosis of the hip remain largely unknown. Adult IgA vasculitis can rarely become complicated by avascular necrosis. We report a case of a 43-year-old male with no significant past medical history who presented with bilateral avascular necrosis of the hips in the setting of acute IgA vasculitis.

Keywords: Vasculitis; IgA Vasculitis; Avascular Necrosis

Abbreviations: IgA vasculitis: Immunoglobulin A Vasculitis; HSP: Henoch-Schönlein Purpura; AVN: Avascular Necrosis

\section{Introduction}

Immunoglobulin A vasculitis (IgA vasculitis), formerly known as Henoch-Schönlein purpura (HSP), is a small vessel vasculitis that is mediated by immune complex deposition in the small vessels associated with activation of leukocytes, leaving IgA deposits in the vessel wall. It usually manifests with renal disease, abdominal pain, arthritis or arthralgia and palpable purpura. Compared to children, IgA vasculitis is uncommon in adults [1]. Although both children and adults experience palpable purpura in the lower extremities, adults more frequently experience purpura in the upper extremities as well. Adults more frequently have manifestations such as elevated C-reactive protein levels, elevated IgA levels, diarrhea, anemia, and more severe renal involvement with worse outcome. Interestingly, adults less frequently experience arthralgia compared to children [2]. Avascular necrosis (AVN), also referred to as osteonecrosis, occurs when vascular supply to subchondral bone is compromised. This leads to the demise of bone marrow and osteocytes, ultimately causing mechanical failure of the joint. The most common affected bone is the femur, due to its unstable blood supply at the head. AVN of the hip is initially asymptomatic; then, it produces a deep pain in the groin radiating to the knee or buttock. As the femoral head is part of a weight bearing joint, activities with weight bearing exacerbate symptoms while rest alleviates them [3]. We describe a unique case of $\operatorname{IgA}$ vasculitis first presented as acute bilateral hip pain followed by palpable purpura of the upper and lower extremities and abdominal pain. Skin biopsy confirmed IgA vasculitis. To our knowledge, this presentation of IgA vasculitis masquerading as AVN has not been commonly reported in the literature.

\section{Case Presentation}

Our patient is a 43-year-old male with no significant past medical history, who presented with bilateral hip pain, predominantly on the right side. He described the pain as dull in nature, gradual in onset, and progressively worsening, associated with limited range of motion of the right hip. A few days later, he developed raised, painful rash over both the upper and the lower extremities with minimal abdominal pain. The patient denied any fever or chills, cough or chest pain, diarrhea or urinary symptoms, and history of trauma or other joint disease. On physical exam, his vital signs 
were stable: blood pressure of 131/88mmHg, heart rate of 81 beats per minute, respiratory rate of 20 breaths per minute, temperature of $36{ }^{\circ} \mathrm{C}$, and oxygen saturation at $96 \%$ on room air. The patient was alert and oriented. On cardiac examination, the patient had a regular rate and rhythm with normal S1 and S2. Respiratory and abdominal examinations showed clear breath sounds bilaterally and mild lower abdominal tenderness. Skin examination was significant for palpable purpura in the lower abdomen, bilateral forearms, and bilateral lower extremities (Figure 1). On musculoskeletal exam, patient had significant limitation in the movement of the right hip in all directions, including abduction, flexion, external and internal rotation.

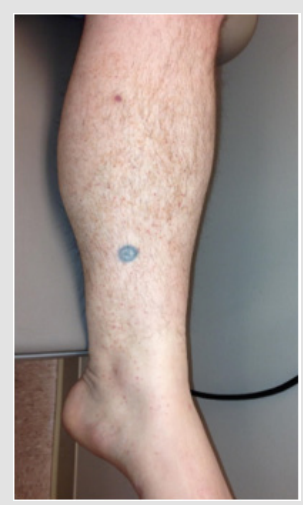

Figure 1: Purpuric Skin rash of the lower extremity.

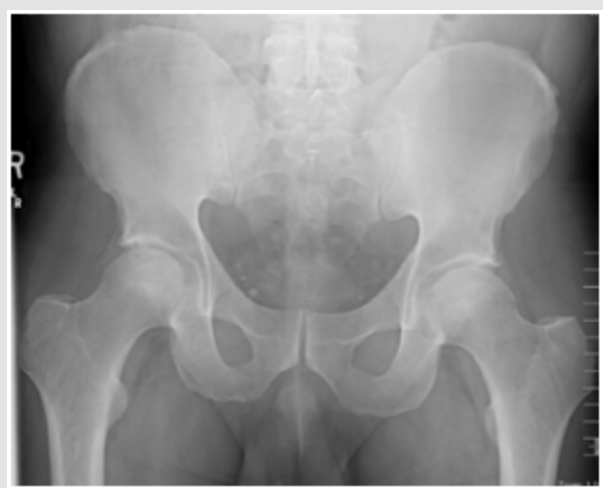

Figure 2: Hip x-ray showing degenerative changes of both hips with subchondral sclerosis.

Patient had guarding and pain with movement. Of note, Log roll, FABER and FADIR tests were positive on the right hip. Left hip had slight limitation of movement. Bilateral hip x-rays showed degenerative changes of both hips with subchondral sclerosis with no acute ossific abnormality (Figure 2). MRI imaging of both hips demonstrated AVN without collapse or superimposed fracture or significant effusion or significant marrow edema on the left side (Figure 3). However, intense marrow edema throughout the right femoral head and neck suggested superimposed subcortical fracture on the right side with only minimal deformity of the articular surface. The patient underwent punch biopsy of the left lower extremity purpura; microscopic examination showed numerous extravasated erythrocytes associated with perivascular neutrophils and focal leukocytoclasia in the superficial dermis, consistent with leukocytoclastic vasculitis. Direct immunofluorescence examination was negative for IgM and IgG. However, it showed granular deposition of IgA and C3 within the walls of few superficial dermal vessels. These findings are consistent with IgA Vasculitis. Blood workup included the following: CBC revealed WBC of 7.6 X10E9/L, Hemoglobin of 15.5g/dL, and Platelets of 149 X10E9/L.

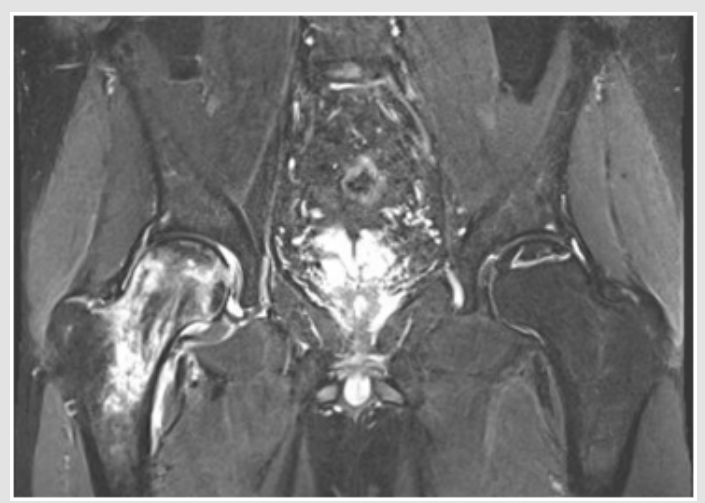

Figure 3: Coronal MRI of the bilateral hip showing evidence for bilateral AVN. There is some bright T2 signal surrounding the infarct at the top of the left femoral head without significant edema to suggest collapse, fragmentation or fracture.

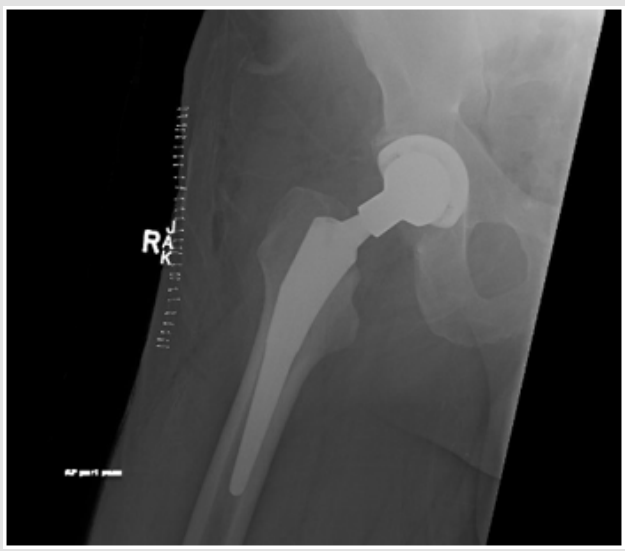

Figure 4: X-ray of the Right hip showing right total hip arthroplasty in appropriate alignment.

Serum creatinine was $1.02 \mathrm{mg} / \mathrm{dL}$. BUN was $19 \mathrm{mg} / \mathrm{dL}$. AST was 37U/L, and ALT was 40U/L. Urinalysis revealed: 0-2 RBCs, 0-2 white blood cells and no proteinuria. ESR was $36 \mathrm{~mm} / \mathrm{h}$. Rheumatoid factor was $<10 \mathrm{IU} / \mathrm{mL}$. ANA was negative. Due to the severe symptoms and the MRI findings of subcortical fracture of the right hip, the patient underwent right total hip replacement (Figure 4). He was treated with a short course of prednisone $0.5 \mathrm{mg} / \mathrm{kg}$ on a tapered dosing regimen for 1 month after the hip replacement. This regimen helped to resolve his skin lesions. However, the 
patient experienced a relapse of the skin lesions 1 week following the discontinuation of his prednisone regimen. He was treated with another course of $0.5 \mathrm{mg} / \mathrm{kg}$ with the addition of methotrexate $15 \mathrm{mg}$ weekly. The new regimen was adequate to resolve his skin lesions.

\section{Discussion}

To our knowledge, avascular necrosis is a rare initial presentation of IgA vasculitis that has not been frequently reported in the literature. AVN as a complication has been reported in a patient with chronic IgA vasculitis [4]. However, this case differs in that AVN was the primary finding followed by the development of classical IgA vasculitis manifestations such as palpable purpura and abdominal pain. Additionally, AVN typically affects one femoral head and both become involved within 2 years [3]. This case presents as bilateral AVN of the hip, though the right side was more affected. When approaching a patient with hip pain, various specialized physical examination maneuvers can be done in order to narrow the differential diagnosis. The FABER test, also known as the Patrick test, is an acronym for the exam maneuver with a patient supine: flexion of the leg at 45 degrees, hip abduction, and external rotation; a positive test occurs when pain is elicited posteriorly in the sacroiliac joint, posterior hip, or lumbar spine. A positive FABER test may also indicate intraarticular arthropathy of the hip if the pain is localized to the groin.

The FADIR test, or impingement test, is another abbreviated maneuver for passive complete flexion, adduction, and internal rotation of the leg with the patient supine; a positive test occurs when pain is elicited. The log roll test, also known as passive supine rotation or the Freiberg test, is another specialized physical exam maneuver conducted with the patient supine. During the log roll test, the patient's leg is extended and relaxed while the clinician internally and externally rotates the leg in a log roll motion. A positive test is when pain or restricted movement of the leg occur [5]. For our patient, the log roll, FABER and FADIR tests were all positive on the right hip. Avascular necrosis can be primary or secondary in etiology. Most common causes of AVN include trauma, glucocorticoid use, and excessive alcohol consumption. The exact pathophysiology of AVN from atraumatic etiology is not yet well understood. However, diagnosis is effective with MRI, the gold standard for AVN diagnosis where sensitivity and specificity reach $99 \%$. MRI remains the imaging modality of choice for early detection and the most effective tool to stage AVN [3]. There are several staging systems for AVN including the Ficat and Arlet Classification System and the Steinberg Staging System. The Ficat and Arlet Classification System is based upon radiographic findings categorized in four stages, with stage I as normal imaging and stage IV as femoral head collapse and osteoarthritis of the hip. According to this system and his imaging, our patient would be categorized as a stage IIA due to the subchondral sclerosis without crescent sign on x-ray.
The Steinberg Staging System elaborates on the first system by categorizing findings into six stages, from normal imaging to advanced degenerative joint disease, with substages based on the intensity of femoral head involvement. Based upon the Steinberg Staging System and his imaging, our patient would be classified as a stage IIB due to the moderate subchondral sclerosis involving between $15-30 \%$ of the femoral head [6]. Initial treatment of avascular necrosis is largely supportive, observation and protection of the joint from bearing weight. The definitive treatment is surgical to either preserve the femoral head or to completely replace the hip via arthroplasty [3]. Immunoglobulin A vasculitis continues to have an unknown etiology. Diagnosis is based upon clinical findings and tissue biopsy with IgA deposits on immunofluorescence. IgA vasculitis also typically receives supportive treatment as the disease tends to be self-limited. Oral glucocorticoids are indicated, though, for severe purpura, edema, or abdominal pain [7]. In this case, our patient initially presented with AVN and concomitantly developed signs and symptoms of IgA vasculitis. He underwent arthroplasty of the right hip and was started on a tapering dose of prednisone. Skin biopsy demonstrated classical features of IgA vasculitis. We propose AVN as a manifestation of systemic vasculitis in this patient due to involvement of both hips, and lack of other offending factors such as use of systemic steroids before the onset of AVN.

\section{Conclusion}

Common symptoms of IgA vasculitis include palpable purpura and abdominal pain. Here, we describe a patient with IgA vasculitis who had an unusual initial presentation of bilateral avascular necrosis of the hips followed by abdominal pain and purpura. The occurrence of AVN in IgA vasculitis is a rare complication; but this does not typically occur during initial presentation or as a bilateral finding. No other causes adequately characterized this patient's condition.

\section{Conflict of Interest}

We do not have any conflicts of interest to disclose. There was no source of funding for this case report.

\section{References}

1. Hocevar A, Ziga Rotar, Vesna Jurčić, Jože Pižem, Saša Čučnik, et al. (2016) IgA vasculitis in adults: The performance of the EULAR/PRINTO/PRES classification criteria in adults. Arthritis Res Ther 18: 58.

2. Kang Y, Jin su Park, You Jung Ha, Mi il Kang, Hee Jin Park, et al. (2014) Differences in clinical manifestations and outcomes between adult and child patients with Henoch-Schonlein purpura. J Korean Med Sci 29(2): 198-203.

3. Baig SA, Baig MN (2018) Osteonecrosis of the Femoral Head: Etiology, Investigations, and Management. Cureus 10(8): e3171.

4. Hirahara K, Kano Y, Asano Y, Shiohara T (2013) Osteonecrosis of the femoral head in a patient with Henoch-Schonlein purpura and druginduced hypersensitivity syndrome treated with corticosteroids. Acta Derm Venereol 93(1): 85-86.

5. Wilson JJ, Furukawa M (2014) Evaluation of the patient with hip pain Am Fam Physician 89(1): 27-34. 
6. Moya Angeler J, Gianakos AL, Villa JC, Ni A, Lane JM (2015) Current concepts on osteonecrosis of the femoral head. World J Orthop 6(8): 590-601.

\section{ISSN: 2574-1241}

DOI: $10.26717 /$ BJSTR.2020.26.004325

Altorok N. Biomed J Sci \& Tech Res

\section{(c) This work is licensed under Creative} Commons Attribution 4.0 License

Submission Link: https://biomedres.us/submit-manuscript.php
7. Jithpratuck W, Yasmin Elshenawy, Hana Saleh, George Youngberg, David S Chi, et al. (2011) The clinical implications of adult-onset henochschonelin purpura. Clin Mol Allergy 9(1): 9

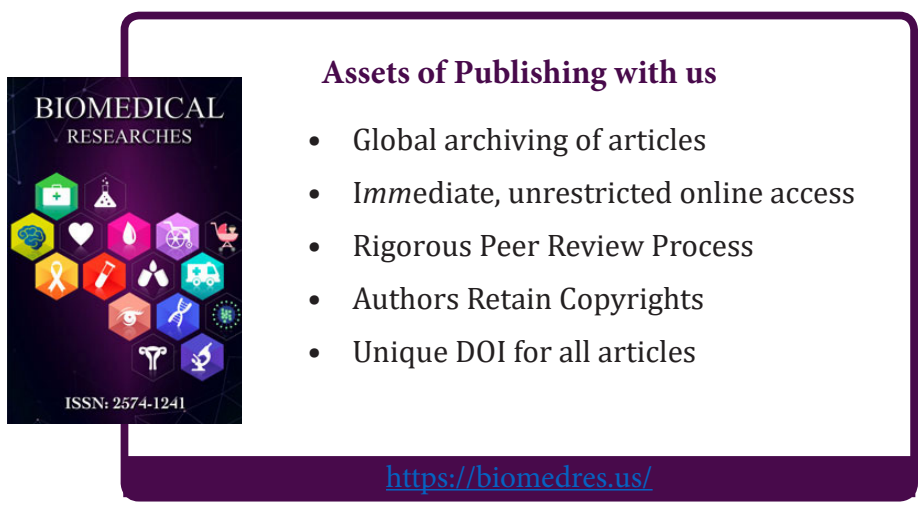

